\title{
ON THE GROUP INVERSE OF LINEAR COMBINATIONS OF TWO GROUP INVERTIBLE MATRICES*
}

\author{
XIAOJI LIU ${ }^{\dagger}$, LINGLING WU ${ }^{\ddagger}$, AND JULIO BENíTEZ
}

\begin{abstract}
In this paper, some formulas are found for the group inverse of $a P+b Q$, where $P$ and $Q$ are two nonzero group invertible complex matrices satisfying certain conditions and $a, b$ nonzero complex numbers.
\end{abstract}

Key words. Group inverse, Linear combination.

AMS subject classifications. 15A09, 15A57.

1. Introduction. Throughout this paper, $\mathbb{C}^{n \times m}$ stands for the set of $n \times m$ complex matrices. $A^{*}$ denotes the conjugate transpose of $A \in \mathbb{C}^{n \times m}$. A matrix $A \in \mathbb{C}^{n \times n}$ is said to be group invertible if there exists $X \in \mathbb{C}^{n \times n}$ such that

$$
A X A=A, \quad X A X=X, \quad A X=X A
$$

hold. If such matrix $X$ exists, then it is unique, denoted by $A^{\#}$, and called the group inverse of $A$. It is well known that the group inverse of a square matrix $A$ exists if and only if $\operatorname{rank}\left(A^{2}\right)=\operatorname{rank}(A)$ (see, for example, [1, Section 4.4] for details). Clearly, not every matrix is group invertible. It is straightforward to prove that $A$ is group invertible if and only if $A^{*}$ is group invertible, and in this case, one has $\left(A^{\#}\right)^{*}=\left(A^{*}\right)^{\#}$. Also, it should be evident that if $A \in \mathbb{C}^{n \times n}$ and $S \in \mathbb{C}^{n \times n}$ is nonsingular, then $A$ is group invertible if and only if $S A S^{-1}$ is group invertible, and in this situation, one has $\left(S A S^{-1}\right)^{\#}=S A^{\#} S^{-1}$.

Recently, some formulas for the Drazin inverse of a sum of two matrices (or two bounded operators in a Banach space) under some conditions were given (see $[4,5,7,8,9,10,11,14]$ and references therein). Let us remark that the group inverse

\footnotetext{
*Received by the editors on November 23, 2010. Accepted for publication on May 8, 2011. Handling Editor: Oskar Maria Baksalary.

${ }^{\dagger}$ College of Mathematics and Computer Science, Guangxi University for Nationalities, Nanning 530006, P.R. China (xiaojiliu72@yahoo.com.cn). Supported by the Guangxi Science Foundation (No. 0832084) and the Guangxi University Innovation Plan (No. gxun-chx2009092).

${ }^{\ddagger}$ College of Mathematics and Computer Science, Guangxi University for Nationalities, Nanning 530006, P.R. China (wulingling-1982@163.com).

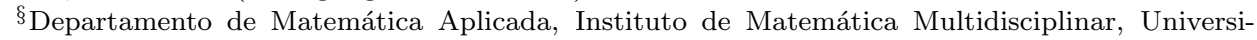
dad Politécnica de Valencia, Valencia, Spain (jbenitez@mat.upv.es). Supported by Spanish Project MTM2010-18539.
} 
is a particular case of the Drazin inverse. Here, we will find sufficient conditions for a linear combination of two group invertible matrices be group invertible, and under these conditions, we will give some expressions for the group inverse of this linear combination. It is noteworthy that Theorem 2.3 given here generalizes $[2$, Theorem $3.1]$.

A previous study of the group invertibility of expressions containing two idempotents was made in [12]. In [12], under the conditions

$$
\begin{aligned}
& (P Q)^{2}=(Q P)^{2} \text { or } \\
& P Q=Q P \text { or } P Q P=Q P Q=P Q \text { or } P Q P=Q P Q \text { or }(P Q)^{2}=0
\end{aligned}
$$

where $P, Q \in \mathbb{C}_{n, n}$ are two distinct nonzero idempotents, the authors studied the group invertibility of expressions of the form $a P+b Q+c P Q+d Q P+e P Q P+$ $f Q P Q+g P Q P Q$, being $a, b, c, d, e, f, g \in \mathbb{C}$. In this present paper, we will study the group invertibility of linear combinations of two group invertible matrices $P, Q \in \mathbb{C}_{n, n}$ satisfying

$$
P Q Q^{\#}=Q P P^{\#} \text { or } P Q Q^{\#}=Q P P^{\#}, Q Q^{\#} P=P P^{\#} Q \text { or } Q P^{\#} P=P .
$$

Let us note that there two main differences between [12] and our paper:

(a) The matrices considered in [12] are idempotents and the matrices considered here are group invertible (observe that if $X \in \mathbb{C}_{n, n}$ is idempotent, then $X$ is group invertible and $\left.X^{\#}=X\right)$. The class of square matrices considered here is strictly greater than the class of idempotent matrices. It is easy to construct nontrivial and interesting examples: it is sufficient to consider a diagonalizable matrix which is not an idempotent.

(b) The conditions (1.1) and (1.2) are quite different. For instance, when we particularize (1.2) for two idempotents $P, Q \in \mathbb{C}_{n, n}$ we get

$$
P Q=Q P \text { or } Q P=P
$$

or if we particularize (1.2) for two tripotent matrices $P, Q \in \mathbb{C}_{n, n}$ we get

$$
P Q^{2}=Q P^{2} \text { or } P Q^{2}=Q P^{2}, Q^{2} P=P^{2} Q \text { or } Q P^{2}=P .
$$

It will be useful to introduce the concept of the spectral idempotent corresponding to 0 ; If $P \in \mathbb{C}^{n \times n}$ is group invertible, we shall denote $P^{\pi}=I_{n}-P P^{\#}$. It is evident that $P^{\pi}$ is an idempotent and $P P^{\pi}=P^{\pi} P=0$. It is obvious that if $P \in \mathbb{C}^{n \times n}$ is group invertible and $c \in \mathbb{C} \backslash\{0\}$, then $P^{\pi}=(c P)^{\pi}$.

2. Some additive results on group inverses. The following two results will be useful in the sequel. 
Theorem 2.1. [13, Exercises 5.10.12 and 5.10.13] Let $A \in \mathbb{C}^{n \times n}$. Then $A$ is a group invertible matrix if and only if there are nonsingular matrices $U \in \mathbb{C}^{n \times n}$ and $C \in \mathbb{C}^{r \times r}$ such that $A=U(C \oplus 0) U^{-1}$, where $r$ is the rank of $A$. In this case, one has $A^{\#}=U\left(C^{-1} \oplus 0\right) U^{-1}$.

Theorem 2.2. [3] Suppose $M=\left[\begin{array}{cc}A & B \\ 0 & C\end{array}\right] \in \mathbb{C}^{n \times n}, A \in \mathbb{C}^{m \times m}$. Then

(i) $M^{\#}$ exists if and only if $A^{\#}$ and $C^{\#}$ exist and $A^{\pi} B C^{\pi}=0$.

(ii) If $M^{\#}$ exists, then $M^{\#}=\left[\begin{array}{cc}A^{\#} & X \\ 0 & C^{\#}\end{array}\right]$, where

$$
X=\left(A^{\#}\right)^{2} B C^{\pi}+A^{\pi} B\left(C^{\#}\right)^{2}-A^{\#} B C^{\#} .
$$

The following result gives a sufficient condition for a linear combination of two group invertible matrices be another group invertible matrix. Let us remark that if $\lambda$ is a nonzero complex number and $A \in \mathbb{C}^{n \times n}$ is group invertible, then $(\lambda A)^{\#}=\lambda^{-1} A^{\#}$, which justifies inclusion of the last statement in Theorem 2.3 since it permits to find the group inverse of $a P+b Q$ when $a+b=0$.

TheOrem 2.3. Let $P, Q \in \mathbb{C}^{n \times n}$ be two group invertible matrices and let $a, b$ be two nonzero complex numbers. If $P Q Q^{\#}=Q P P^{\#}$, then $a P+b Q$ is group invertible. If $a+b \neq 0$, then

$$
\begin{aligned}
& (a P+b Q)^{\#} \\
& =\frac{1}{a+b}\left[P^{\#}+Q^{\#}-P^{\#} Q Q^{\#}\right]+\left(\frac{1}{a}-\frac{1}{a+b}\right) Q^{\pi} P^{\#}+\left(\frac{1}{b}-\frac{1}{a+b}\right) P^{\pi} Q^{\#} .
\end{aligned}
$$

Moreover,

$$
(P-Q)^{\#}=(P-Q)\left(P^{\#}-Q^{\#}\right)^{2}
$$

Proof. Let $r$ be the rank of $P$. Since $P$ is group invertible, there are nonsingular matrices $U \in \mathbb{C}^{n \times n}$ and $A \in \mathbb{C}^{r \times r}$ such that

$$
P=U(A \oplus 0) U^{-1} \text {. }
$$

Let us write

$$
Q=U\left[\begin{array}{ll}
Q_{1} & Q_{2} \\
Q_{3} & Q_{4}
\end{array}\right] U^{-1}, \quad Q Q^{\#}=U\left[\begin{array}{ll}
X_{1} & X_{2} \\
X_{3} & X_{4}
\end{array}\right] U^{-1}, \quad Q_{1}, X_{1} \in \mathbb{C}^{r \times r} .
$$

Since

$$
P Q Q^{\#}=U\left[\begin{array}{cc}
A X_{1} & A X_{2} \\
0 & 0
\end{array}\right] U^{-1} \quad \text { and } \quad Q P P^{\#}=U\left[\begin{array}{ll}
Q_{1} & 0 \\
Q_{3} & 0
\end{array}\right] U^{-1}
$$


from the hypothesis and the nonsingularity of $A$, we get

$$
X_{2}=0, \quad Q_{3}=0, \quad A X_{1}=Q_{1} .
$$

Since

$$
Q=U\left[\begin{array}{cc}
Q_{1} & Q_{2} \\
0 & Q_{4}
\end{array}\right] U^{-1}
$$

by applying Theorem 2.2 , we have that $Q_{1}$ and $Q_{4}$ are group invertible and

$$
Q^{\#}=U\left[\begin{array}{cc}
Q_{1}^{\#} & M \\
0 & Q_{4}^{\#}
\end{array}\right] U^{-1},
$$

where $M$ is some matrix in $\mathbb{C}^{r \times(n-r)}$ whose explicit form will be unnecessary. Using (2.4), (2.5), and the representation of $Q Q^{\#}$ in (2.2), we get

$$
X_{1}=Q_{1} Q_{1}^{\#}, \quad X_{3}=0, \quad X_{4}=Q_{4} Q_{4}^{\#} .
$$

Using the representation of $Q Q^{\#}$ in (2.2), the first equality of (2.3), and (2.6) we get

$$
Q Q^{\#}=U\left[\begin{array}{cc}
Q_{1} Q_{1}^{\#} & 0 \\
0 & Q_{4} Q_{4}^{\#}
\end{array}\right] U^{-1} .
$$

Using the equalities $Q=Q\left(Q Q^{\#}\right)=\left(Q Q^{\#}\right) Q$ and the representations of $Q$ and $Q Q^{\#}$ written in (2.4) and (2.7), respectively, we get

$$
Q_{2}=Q_{2} Q_{4} Q_{4}^{\#}=Q_{1} Q_{1}^{\#} Q_{2}
$$

Let $x$ and $y$ be the rank of $Q_{1}$ and $Q_{4}$, respectively. Since $Q_{1}$ and $Q_{4}$ are group invertible matrices, there exist nonsingular matrices $W \in \mathbb{C}^{r \times r}, B_{1} \in \mathbb{C}^{x \times x}, V \in$ $\mathbb{C}^{(n-r) \times(n-r)}$, and $B_{2} \in \mathbb{C}^{y \times y}$ such that

$$
Q_{1}=W\left(B_{1} \oplus 0\right) W^{-1}, \quad Q_{4}=V\left(B_{2} \oplus 0\right) V^{-1} .
$$

Let us write $Q_{2} \in \mathbb{C}^{r \times(n-r)}$ as follows:

$$
Q_{2}=W\left[\begin{array}{ll}
B_{3} & B_{4} \\
B_{5} & B_{6}
\end{array}\right] V^{-1}, \quad B_{3} \in \mathbb{C}^{x \times y} .
$$

From $Q_{2}=Q_{1} Q_{1}^{\#} Q_{2},(2.8)$, and (2.9) we get $B_{5}=0$ and $B_{6}=0$. Using $Q_{2}=$ $Q_{2} Q_{4} Q_{4}^{\#},(2.8)$, and (2.9) leads to $B_{4}=0$. Hence,

$$
Q_{2}=W\left[\begin{array}{cc}
B_{3} & 0 \\
0 & 0
\end{array}\right] V^{-1}
$$


Now, let us write $A$ as follows

$$
A=W\left[\begin{array}{ll}
A_{1} & A_{2} \\
A_{3} & A_{4}
\end{array}\right] W^{-1}, \quad A_{1} \in \mathbb{C}^{x \times x} .
$$

From the last equality of (2.3), the first one of (2.6), the representation of $Q_{1}$ in (2.8) and the representation of $A$ in $(2.11)$ we get

$$
A_{1}=B_{1}, \quad A_{3}=0 .
$$

The representation (2.11), $A_{3}=0$, and the nonsingularity of $A$ yield that $A_{1}$ and $A_{4}$ are nonsingular (in fact, we have previously obtained $A_{1}=B_{1}$, which is another way to prove the nonsingularity of $A_{1}$ ) and

$$
A^{-1}=W\left[\begin{array}{cc}
A_{1}^{-1} & -A_{1}^{-1} A_{2} A_{4}^{-1} \\
0 & A_{4}^{-1}
\end{array}\right] W^{-1} .
$$

Let us define the nonsingular matrix $Z=U(W \oplus V)$. From (2.1) and (2.4), we obtain

$$
P=Z\left[\begin{array}{cc}
W^{-1} A W & 0 \\
0 & 0
\end{array}\right] Z^{-1}, \quad Q=Z\left[\begin{array}{cc}
W^{-1} Q_{1} W & W^{-1} Q_{2} V \\
0 & V^{-1} Q_{4} V
\end{array}\right] Z^{-1}
$$

Now we use (2.11), (2.12), (2.8), and (2.10):

$$
P=Z\left[\begin{array}{cccc}
B_{1} & A_{2} & 0 & 0 \\
0 & A_{4} & 0 & 0 \\
0 & 0 & 0 & 0 \\
0 & 0 & 0 & 0
\end{array}\right] Z^{-1}, \quad Q=Z\left[\begin{array}{cccc}
B_{1} & 0 & B_{3} & 0 \\
0 & 0 & 0 & 0 \\
0 & 0 & B_{2} & 0 \\
0 & 0 & 0 & 0
\end{array}\right] Z^{-1}
$$

and thus

$$
a P+b Q=Z\left[\begin{array}{cccc}
(a+b) B_{1} & a A_{2} & b B_{3} & 0 \\
0 & a A_{4} & 0 & 0 \\
0 & 0 & b B_{2} & 0 \\
0 & 0 & 0 & 0
\end{array}\right] Z^{-1}
$$

As is easy to see from the definition of group inverse and the representations given in (2.13)

$$
P^{\#}=Z\left[\begin{array}{cccc}
B_{1}^{-1} & -B_{1}^{-1} A_{2} A_{4}^{-1} & 0 & 0 \\
0 & A_{4}^{-1} & 0 & 0 \\
0 & 0 & 0 & 0 \\
0 & 0 & 0 & 0
\end{array}\right] Z^{-1}
$$

and

$$
Q^{\#}=Z\left[\begin{array}{cccc}
B_{1}^{-1} & 0 & -B_{1}^{-1} B_{3} B_{2}^{-1} & 0 \\
0 & 0 & 0 & 0 \\
0 & 0 & B_{2}^{-1} & 0 \\
0 & 0 & 0 & 0
\end{array}\right] Z^{-1}
$$


Moreover, a representation for $P P^{\#}$ and $Q Q^{\#}$ will be useful. From (2.1) and the definition of $Z$ we get

$$
P P^{\#}=U\left(I_{r} \oplus 0\right) U^{-1}=Z\left(W^{-1} \oplus V^{-1}\right)\left(I_{r} \oplus 0\right)(W \oplus V) Z^{-1}=Z\left(I_{r} \oplus 0\right) Z^{-1} .
$$

Hence,

$$
P P^{\#}=Z\left(I_{x} \oplus I_{r-x} \oplus 0 \oplus 0\right) Z^{-1} .
$$

From (2.7) and (2.8) we obtain

$$
Q Q^{\#}=U\left(Q_{1} Q_{1}^{\#} \oplus Q_{4} Q_{4}^{\#}\right) U^{-1}=Z\left(I_{x} \oplus 0 \oplus I_{y} \oplus 0\right) Z^{-1}
$$

Assume that $a+b \neq 0$. It is not hard to see that the matrix

$$
X=Z\left[\begin{array}{cccc}
(a+b)^{-1} B_{1}^{-1} & -(a+b)^{-1} B_{1}^{-1} A_{2} A_{4}^{-1} & -(a+b)^{-1} B_{1}^{-1} B_{3} B_{2}^{-1} & 0 \\
0 & a^{-1} A_{4}^{-1} & 0 & 0 \\
0 & 0 & b^{-1} B_{2}^{-1} & 0 \\
0 & 0 & 0 & 0
\end{array}\right] Z^{-1}
$$

satisfies $(a P+b Q) X=X(a P+b Q),(a P+b Q) X(a P+b Q)=a P+b Q$, and $X(a P+$ $b Q) X=X$. Therefore, $a P+b Q$ is group invertible and $(a P+b Q)^{\#}=X$. We shall express $X$ in terms of expressions containing matrices $P$ and $Q$. To this end, let us observe that

$$
\begin{gathered}
X=\frac{1}{a+b} Z\left[\begin{array}{cccc}
B_{1}^{-1} & -B_{1}^{-1} A_{2} A_{4}^{-1} & -B_{1}^{-1} B_{3} B_{2}^{-1} & 0 \\
0 & 0 & 0 & 0 \\
0 & 0 & 0 & 0 \\
0 & 0 & 0 & 0
\end{array}\right] Z^{-1}+ \\
+\frac{1}{a} Z\left[\begin{array}{cccc}
0 & 0 & 0 & 0 \\
0 & A_{4}^{-1} & 0 & 0 \\
0 & 0 & 0 & 0 \\
0 & 0 & 0 & 0
\end{array}\right] Z^{-1}+\frac{1}{b} Z\left[\begin{array}{cccc}
0 & 0 & 0 & 0 \\
0 & 0 & 0 & 0 \\
0 & 0 & B_{2}^{-1} & 0 \\
0 & 0 & 0 & 0
\end{array}\right] Z^{-1} .
\end{gathered}
$$

We compute $Q^{\pi} P^{\#}$ and $P^{\pi} Q^{\#}$. By using (2.14) and (2.17) we get

$$
\begin{aligned}
Q^{\pi} P^{\#} & =Z\left[\begin{array}{cccc}
0 & 0 & 0 & 0 \\
0 & I_{r-x} & 0 & 0 \\
0 & 0 & 0 & 0 \\
0 & 0 & 0 & I_{n-r-y}
\end{array}\right]\left[\begin{array}{cccc}
B_{1}^{-1} & -B_{1}^{-1} A_{2} B_{4}^{-1} & 0 & 0 \\
0 & A_{4}^{-1} & 0 & 0 \\
0 & 0 & 0 & 0 \\
0 & 0 & 0 & 0
\end{array}\right] Z^{-1} \\
& =Z\left[\begin{array}{cccc}
0 & 0 & 0 & 0 \\
0 & A_{4}^{-1} & 0 & 0 \\
0 & 0 & 0 & 0 \\
0 & 0 & 0 & 0
\end{array}\right] Z^{-1}
\end{aligned}
$$


By using (2.15) and (2.16) we obtain

$$
\begin{aligned}
P^{\pi} Q^{\#} & =Z\left[\begin{array}{cccc}
0 & 0 & 0 & 0 \\
0 & 0 & 0 & 0 \\
0 & 0 & I_{y} & 0 \\
0 & 0 & 0 & I_{n-r-y}
\end{array}\right]\left[\begin{array}{cccc}
B_{1}^{-1} & 0 & -B_{1}^{-1} B_{3} B_{2}^{-1} & 0 \\
0 & 0 & 0 & 0 \\
0 & 0 & B_{2}^{-1} & 0 \\
0 & 0 & 0 & 0
\end{array}\right] Z^{-1} \\
& =Z\left[\begin{array}{cccc}
0 & 0 & 0 & 0 \\
0 & 0 & 0 & 0 \\
0 & 0 & B_{2}^{-1} & 0 \\
0 & 0 & 0 & 0
\end{array}\right] Z^{-1} .
\end{aligned}
$$

Now, we shall compute $P^{\#} Q Q^{\#}$ :

$$
\begin{aligned}
& P^{\#} Q Q^{\#}=Z\left[\begin{array}{cccc}
B_{1}^{-1} & -B_{1}^{-1} A_{2} B_{4}^{-1} & 0 & 0 \\
0 & A_{4}^{-1} & 0 & 0 \\
0 & 0 & 0 & 0 \\
0 & 0 & 0 & 0
\end{array}\right]\left[\begin{array}{cccc}
I_{x} & 0 & 0 & 0 \\
0 & 0 & 0 & 0 \\
0 & 0 & I_{y} & 0 \\
0 & 0 & 0 & 0
\end{array}\right] Z^{-1} \\
& =Z\left[\begin{array}{cccc}
B_{1}^{-1} & 0 & 0 & 0 \\
0 & 0 & 0 & 0 \\
0 & 0 & 0 & 0 \\
0 & 0 & 0 & 0
\end{array}\right] Z^{-1}
\end{aligned}
$$

parenthetically, we can prove that $P^{\#} Q Q^{\#}=Q^{\#} P P^{\#}$. Therefore,

$$
X=\frac{1}{a+b}\left[P^{\#}+Q^{\#}-P^{\#} Q Q^{\#}\right]+\left(\frac{1}{a}-\frac{1}{a+b}\right) Q^{\pi} P^{\#}+\left(\frac{1}{b}-\frac{1}{a+b}\right) P^{\pi} Q^{\#} .
$$

On the other hand, we will prove that $P-Q$ is group invertible and we will find an expression for $(P-Q)^{\#}$. From $(2.13)$ we have

$$
P-Q=Z\left[\begin{array}{cccc}
0 & A_{2} & -B_{3} & 0 \\
0 & A_{4} & 0 & 0 \\
0 & 0 & -B_{2} & 0 \\
0 & 0 & 0 & 0
\end{array}\right] Z^{-1}
$$

It is not hard to see that

$$
Y=Z\left[\begin{array}{cccc}
0 & A_{2}\left(A_{4}^{-1}\right)^{2} & -B_{3}\left(B_{2}^{-1}\right)^{2} & 0 \\
0 & A_{4}^{-1} & 0 & 0 \\
0 & 0 & -B_{2}^{-1} & 0 \\
0 & 0 & 0 & 0
\end{array}\right] Z^{-1}
$$

satisfies $(P-Q) Y=Y(P-Q),(P-Q) Y(P-Q)=P-Q$, and $Y(P-Q) Y=Y$. Therefore, $P-Q$ is group invertible and $(P-Q)^{\#}=Y$. We shall express $Y$ in terms 
of expressions containing matrices $P$ and $Q$. We compute $P^{\#}-Q^{\#}$. By using (2.14) and (2.15) we get

$$
P^{\#}-Q^{\#}=Z\left[\begin{array}{cccc}
0 & -B_{1}^{-1} A_{2} A_{4}^{-1} & B_{1}^{-1} B_{3} B_{2}^{-1} & 0 \\
0 & A_{4}^{-1} & 0 & 0 \\
0 & 0 & -B_{2}^{-1} & 0 \\
0 & 0 & 0 & 0
\end{array}\right] Z^{-1}
$$

Now, by using (2.21) we compute

$$
\left(P^{\#}-Q^{\#}\right)^{2}=Z\left[\begin{array}{cccc}
0 & -B_{1}^{-1} A_{2}\left(A_{4}^{-1}\right)^{2} & -B_{1}^{-1} B_{3}\left(B_{2}^{-1}\right)^{2} & 0 \\
0 & \left(A_{4}^{-1}\right)^{2} & 0 & 0 \\
0 & 0 & \left(B_{2}^{-1}\right)^{2} & 0 \\
0 & 0 & 0 & 0
\end{array}\right] Z^{-1} .
$$

So, we have

$$
(P-Q)\left(P^{\#}-Q^{\#}\right)^{2}=Z\left[\begin{array}{cccc}
0 & A_{2}\left(A_{4}^{-1}\right)^{2} & -B_{3}\left(B_{2}^{-1}\right)^{2} & 0 \\
0 & A_{4}^{-1} & 0 & 0 \\
0 & 0 & -B_{2}^{-1} & 0 \\
0 & 0 & 0 & 0
\end{array}\right] Z^{-1}=Y .
$$

This finishes the proof.

TheOREM 2.4. Let $P, Q \in \mathbb{C}^{n \times n}$ be two group invertible matrices and let $a, b$ be two nonzero complex numbers. If $Q Q^{\#} P=P P^{\#} Q$, then $a P+b Q$ is group invertible. If $a+b \neq 0$, then

$$
\begin{aligned}
& (a P+b Q)^{\#}= \\
& \frac{1}{a+b}\left[P^{\#}+Q^{\#}-Q^{\#} Q P^{\#}\right]+\left(\frac{1}{a}-\frac{1}{a+b}\right) P^{\#} Q^{\pi}+\left(\frac{1}{b}-\frac{1}{a+b}\right) Q^{\#} P^{\pi} .
\end{aligned}
$$

Moreover,

$$
(P-Q)^{\#}=\left(P^{\#}-Q^{\#}\right)^{2}(P-Q) .
$$

Proof. It is enough to apply Theorem 2.3 for the linear combination $\bar{a} P^{*}+\bar{b} Q^{*}$ and using that a matrix $C$ has group inverse if and only if $C^{*}$ is group invertible and in this case one has $\left(C^{*}\right)^{\#}=\left(C^{\#}\right)^{*}$.

Corollary 2.5. Let $P, Q \in \mathbb{C}^{n \times n}$ be two group invertible matrices and let $a, b$ be two nonzero complex numbers. If $P Q Q^{\#}=Q P P^{\#}$ and $Q Q^{\#} P=P P^{\#} Q$, then $a P+b Q$ and $P Q$ are group invertible. If $a+b \neq 0$, then

$$
(a P+b Q)^{\#}=\frac{1}{a+b} P^{\#} Q Q^{\#}+\frac{1}{a} Q^{\pi} P^{\#}+\frac{1}{b} P^{\pi} Q^{\#} .
$$


Moreover,

$$
(P-Q)^{\#}=P^{\#}-Q^{\#}
$$

and

$$
(P Q)^{\#}=\left(P^{\#} Q Q^{\#}\right)^{2}=(Q P)^{\#}
$$

Proof. Since $P Q Q^{\#}=Q P P^{\#}$, by following the proof of Theorem 2.3 we get that $P$ and $Q$ can be written as in (2.13), $P P^{\#}$ as in (2.16), and $Q Q^{\#}$ as in (2.17). From $Q Q^{\#} P=P P^{\#} Q$ we get $A_{2}=0$ and $B_{3}=0$. Hence,

$$
P=Z\left(B_{1} \oplus A_{4} \oplus 0 \oplus 0\right) Z^{-1}, \quad Q=Z\left(B_{1} \oplus 0 \oplus B_{2} \oplus 0\right) Z^{-1} .
$$

Therefore,

$$
a P+b Q=Z\left((a+b) B_{1} \oplus a A_{4} \oplus b B_{2} \oplus 0\right) Z^{-1}, \quad P Q=Z\left(B_{1}^{2} \oplus 0 \oplus 0 \oplus 0\right) Z^{-1}
$$

and thus $a P+b Q$ and $P Q$ are group invertible and

$$
\begin{gathered}
(a P+b Q)^{\#}=Z\left((a+b)^{\#} B_{1}^{-1} \oplus \frac{1}{a} A_{4}^{-1} \oplus \frac{1}{b} B_{2}^{-1} \oplus 0\right) Z^{-1}, \\
(P Q)^{\#}=Z\left(B_{1}^{-2} \oplus 0 \oplus 0 \oplus 0\right) Z^{-1},
\end{gathered}
$$

where we have denoted $\lambda^{\#}=\lambda^{-1}$ for $\lambda \in \mathbb{C} \backslash\{0\}$ and $0^{\#}=0$. Now, the proof should be easy to be finished by taking into account (2.18), (2.19), and (2.20).

If $P$ and $Q$ are idempotent matrices, then $P$ and $Q$ are group invertible, $P^{\#}=P$ and $Q^{\#}=Q$. By using Theorem 2.3 we recover the following result:

Corollary 2.6. [6, Theorem 3.4] Let $P, Q \in \mathbb{C}^{n \times n}$ be two idempotent matrices such that $P Q=Q P$, then

$$
(P+Q)^{\#}=P+Q-\frac{3}{2} P Q, \quad(P-Q)^{\#}=P-Q
$$

If $P$ and $Q$ are tripotent matrices, then $P$ and $Q$ are group invertible, $P^{\#}=P$ and $Q^{\#}=Q$. By using Theorem 2.3 we get the following corollary.

Corollary 2.7. Let $P, Q \in \mathbb{C}^{n \times n}$ be two tripotent matrices such that $P Q^{2}=$ $Q P^{2}$, then

$$
(P+Q)^{\#}=P+Q-\frac{1}{2} P Q^{2}-\frac{1}{2} P^{2} Q-\frac{1}{2} Q^{2} P
$$


and

$$
(P-Q)^{\#}=(P-Q)^{3}=(P-Q)+\left(Q^{2} P-P^{2} Q\right)+(Q P Q-P Q P)
$$

By using Theorem 2.4, we get the following corollary.

Corollary 2.8. Let $P, Q \in \mathbb{C}^{n \times n}$ be two tripotent matrices such that $P^{2} Q=$ $Q^{2} P$, then

$$
(P+Q)^{\#}=P+Q-\frac{1}{2} P Q^{2}-\frac{1}{2} P^{2} Q-\frac{1}{2} Q P^{2}
$$

and

$$
(P-Q)^{\#}=(P-Q)^{3}=(P-Q)+\left(Q P^{2}-P Q^{2}\right)+(Q P Q-P Q P) .
$$

When $P, Q \in \mathbb{C}^{n \times n}$ are idempotents, the condition $Q P=P$ has been studied many times and it appears in many different fields of applied mathematics (since it can be proved that $Q P=P$ if and only if the range of $P$ is a subspace of the range of $Q$ ). In [6, Corollary 3.2] the authors gave a formula for $(p+q)^{\#}$ when $p$ and $q$ are idempotents in a complex Banach algebra with unity. Here we remove the idempotency condition working in the setting of square complex matrices.

TheOrem 2.9. Let $P, Q \in \mathbb{C}^{n \times n}$ be group invertible. If $Q P^{\#} P=P$ and $a, b \in$ $\mathbb{C} \backslash\{0\}$ satisfy $a+b \neq 0$, then $a P+b Q$ is group invertible and

$$
(a P+b Q)^{\#}=\frac{a}{(a+b)^{2}} P^{\#}+\frac{b}{(a+b)^{2}} Q^{\#}+\frac{a^{2}+2 a b}{b(a+b)^{2}} P^{\pi} Q^{\#} P^{\pi}-\frac{a}{(a+b)^{2}} P^{\#}(Q-P) Q^{\#} .
$$

Proof. Let $r$ be the rank of $P$. By Theorem 2.1, there are nonsingular matrices $U \in \mathbb{C}^{n \times n}$ and $A \in \mathbb{C}^{r \times r}$ such that $P=U(A \oplus 0) U^{-1}$, and therefore, $P^{\#}=U\left(A^{-1} \oplus\right.$ $0) U^{-1}$ and $P^{\pi}=U\left(0 \oplus I_{n-r}\right) U^{-1}$. Let us write

$$
Q=U\left[\begin{array}{ll}
Q_{1} & Q_{2} \\
Q_{3} & Q_{4}
\end{array}\right] U^{-1}
$$

From $Q P P^{\#}=P$ we get $Q_{1}=A$ and $Q_{3}=0$. Thus, we can apply Theorem 2.2 for the group invertible matrix $Q$ obtaining that $Q_{4}$ is group invertible, and

$$
Q^{\#}=U\left[\begin{array}{cc}
A^{-1} & X \\
0 & Q_{4}^{\#}
\end{array}\right] U^{-1}, \quad X=A^{-2} Q_{2} Q_{4}^{\pi}-A^{-1} Q_{2} Q_{4}^{\#} .
$$


Since

$$
a P+b Q=U\left[\begin{array}{cc}
(a+b) A & b Q_{2} \\
0 & b Q_{4}
\end{array}\right] U^{-1}
$$

by using again Theorem 2.2, we get the existence of $(a P+b Q)^{\#}$ and

$$
(a P+b Q)^{\#}=U\left[\begin{array}{cc}
(a+b)^{-1} A^{-1} & Y \\
0 & b^{-1} Q_{4}^{\#}
\end{array}\right] U^{-1}
$$

where

$$
\begin{aligned}
Y & =(a+b)^{-2} A^{-2}\left(b Q_{2}\right)\left(b Q_{4}\right)^{\pi}-(a+b)^{-1} A^{-1}\left(b Q_{2}\right)\left(b Q_{4}\right)^{\#} \\
& =b(a+b)^{-2} A^{-2} Q_{2} Q_{4}^{\pi}-(a+b)^{-1} A^{-1} Q_{2} Q_{4}^{\#} .
\end{aligned}
$$

Now,

$$
U^{-1}\left[\begin{array}{cc}
(a+b)^{-1} A^{-1} & Y \\
0 & b^{-1} Q_{4}^{\#}
\end{array}\right] U=\frac{1}{a+b} P^{\#}+\frac{1}{b} P^{\pi} Q^{\#} P^{\pi}+U^{-1}\left[\begin{array}{cc}
0 & Y \\
0 & 0
\end{array}\right] U
$$

But,

$$
\begin{aligned}
{\left[\begin{array}{cc}
0 & Y \\
0 & 0
\end{array}\right]=} & \frac{b}{(a+b)^{2}}\left[\begin{array}{cc}
0 & A^{-2} Q_{2} Q_{4}^{\pi} \\
0 & 0
\end{array}\right]-\frac{1}{a+b}\left[\begin{array}{cc}
0 & A^{-1} Q_{2} Q_{4}^{\#} \\
0 & 0
\end{array}\right] \\
= & \frac{b}{(a+b)^{2}}\left[\begin{array}{cc}
A^{-1} & A^{-2} Q_{2} Q_{4}^{\pi}-A^{-1} Q_{2} Q_{4}^{\#} \\
0 & Q_{4}^{\#}
\end{array}\right]-\frac{b}{(a+b)^{2}}\left[\begin{array}{cc}
A^{-1} & 0 \\
0 & 0
\end{array}\right] \\
& -\frac{b}{(a+b)^{2}}\left[\begin{array}{cc}
0 & 0 \\
0 & Q_{4}^{\#}
\end{array}\right]+\left(\frac{b}{(a+b)^{2}}-\frac{1}{a+b}\right)\left[\begin{array}{cc}
0 & A^{-1} Q_{2} Q_{4}^{\#} \\
0 & 0
\end{array}\right] \\
= & U\left\{\frac{b}{(a+b)^{2}}\left(Q^{\#}-P^{\#}-P^{\pi} Q^{\#} P^{\pi}\right)+\right. \\
& \left.\left(\frac{b}{(a+b)^{2}}-\frac{1}{a+b}\right) P^{\#}(Q-P) Q^{\#}\right\} U^{-1} .
\end{aligned}
$$

After doing a little algebra we have

$$
\begin{aligned}
& U^{-1}\left[\begin{array}{cc}
(a+b)^{-1} A^{-1} & Y \\
0 & b^{-1} Q_{4}^{\#}
\end{array}\right] U \\
& =\frac{a}{(a+b)^{2}} P^{\#}+\frac{b}{(a+b)^{2}} Q^{\#}+\frac{a^{2}+2 a b}{b(a+b)^{2}} P^{\pi} Q^{\#} P^{\pi}-\frac{a}{(a+b)^{2}} P^{\#}(Q-P) Q^{\#} .
\end{aligned}
$$

This proves the theorem. $\square$ 
3. Group invertibility of a linear combination of two commuting group invertible matrices. The following results are concerned with the group invertibility of a linear combination of two commuting group invertible matrices. Theorem 3.1 below, which is important on its own, permits us to deal with two commuting group invertible matrices in a very simple form. In particular, Theorem 3.1 will allow us to manage linear combinations of two commuting group invertible matrices.

TheOREM 3.1. Let $P, Q \in \mathbb{C}^{n \times n}$ be two group invertible matrices such that $P Q=Q P$, then there are nonsingular matrices $Z \in \mathbb{C}^{n \times n}, A_{1}, B_{1} \in \mathbb{C}^{x \times x}, A_{2} \in$ $\mathbb{C}^{(r-x) \times(r-x)}$, and $B_{2} \in \mathbb{C}^{y \times y}$ such that

$$
P=Z\left(A_{1} \oplus A_{2} \oplus 0 \oplus 0\right) Z^{-1}, \quad Q=Z\left(B_{1} \oplus 0 \oplus B_{2} \oplus 0\right) Z^{-1}, \quad A_{1} B_{1}=B_{1} A_{1} .
$$

Proof. Let $r$ be the rank of $P$. Since $P$ is group invertible, by applying Lemma 2.1, there are nonsingular matrices $U \in \mathbb{C}^{n \times n}$ and $A \in \mathbb{C}^{r \times r}$ such that $P=U(A \oplus 0) U^{-1}$. Let us write

$$
Q=U\left[\begin{array}{ll}
Q_{1} & Q_{2} \\
Q_{3} & Q_{4}
\end{array}\right] U^{-1}
$$

Since

$$
Q P=U\left[\begin{array}{ll}
Q_{1} A & 0 \\
Q_{3} A & 0
\end{array}\right] U^{-1}, \quad P Q=U\left[\begin{array}{cc}
A Q_{1} & A Q_{2} \\
0 & 0
\end{array}\right] U^{-1}, \quad P Q=Q P,
$$

from the nonsingularity of $A$, we get $Q_{1} A=A Q_{1}, Q_{2}=0$, and $Q_{3}=0$. Since $Q=U\left(Q_{1} \oplus Q_{4}\right) U^{-1}$, by applying Theorem 2.2 we get that $Q_{1}$ and $Q_{4}$ are group invertible. Let $x$ and $y$ be the rank of $Q_{1}$ and $Q_{4}$, respectively. By Theorem 2.1, there exist nonsingular matrices $B_{1} \in \mathbb{C}^{x \times x}, W \in \mathbb{C}^{r \times r}, B_{2} \in \mathbb{C}^{y \times y}, V \in \mathbb{C}^{(n-r) \times(n-r)}$ such that $Q_{1}=W\left(B_{1} \oplus 0\right) W^{-1}$ and $Q_{4}=V\left(B_{2} \oplus 0\right) V^{-1}$. If we denote $Z=U(W \oplus V)$, we have $Q=Z\left(B_{1} \oplus 0 \oplus B_{2} \oplus 0\right) Z^{-1}$ and

$$
\begin{aligned}
P & =U(A \oplus 0) U^{-1} \\
& =U\left[\begin{array}{cc}
W & 0 \\
0 & V
\end{array}\right]\left[\begin{array}{cc}
W^{-1} A W & 0 \\
0 & 0
\end{array}\right]\left[\begin{array}{cc}
W^{-1} & 0 \\
0 & V^{-1}
\end{array}\right] U^{-1} \\
& =Z\left(W^{-1} A W \oplus 0\right) Z^{-1} .
\end{aligned}
$$

Let us write

$$
W^{-1} A W=\left[\begin{array}{cc}
X_{1} & X_{2} \\
X_{3} & X_{4}
\end{array}\right], \quad X_{1} \in \mathbb{C}^{x \times x} .
$$

From $A Q_{1}=Q_{1} A$ we get

$$
\left[\begin{array}{ll}
X_{1} & X_{2} \\
X_{3} & X_{4}
\end{array}\right]\left[\begin{array}{cc}
B_{1} & 0 \\
0 & 0
\end{array}\right]=\left[\begin{array}{cc}
B_{1} & 0 \\
0 & 0
\end{array}\right]\left[\begin{array}{ll}
X_{1} & X_{2} \\
X_{3} & X_{4}
\end{array}\right]
$$


which, having in mind the nonsingularity of $B_{1}$, entails $X_{1} B_{1}=B_{1} X_{1}, X_{2}=0$, and $X_{3}=0$. In order to prove the theorem, it is enough to prove that $X_{1}$ and $X_{4}$ are nonsingular; but this follows from $W^{-1} A W=X_{1} \oplus X_{4}$ and the nonsingularity of $A$.

Corollary 3.2. Let $P, Q \in \mathbb{C}^{n \times n}$ be two group invertible matrices such that $P Q=Q P$. Then the algebra generated by $P, Q, P^{\#}$, and $Q^{\#}$ is commutative.

Proof. It follows from Theorem 3.1.

Corollary 3.3. Let $P, Q \in \mathbb{C}^{n \times n}$ be two group invertible matrices such that $P Q=Q P$ and $a, b \in \mathbb{C} \backslash\{0\}$. Then $a P+b Q$ is group invertible if and only if $a P Q Q^{\#}+b Q P P^{\#}$ is group invertible. In this situation one has

$$
(a P+b Q)^{\#}=\left(a P Q Q^{\#}+b Q P P^{\#}\right)^{\#}+\frac{1}{a} P^{\#} Q^{\pi}+\frac{1}{b} Q^{\#} P^{\pi}
$$

Proof. By using the representation (3.1) we have

$$
a P+b Q=Z\left(\left(a A_{1}+b B_{1}\right) \oplus a A_{2} \oplus b B_{2} \oplus 0\right) Z^{-1}
$$

and

$$
a P Q Q^{\#}+b Q P P^{\#}=Z\left(a A_{1}+b B_{1} \oplus 0 \oplus 0 \oplus 0\right) Z^{-1}
$$

Since $A_{1}$ and $B_{1}$ are nonsingular, we have that $a P+b Q$ is group invertible if and only if $a P Q Q^{\#}+b Q P P^{\#}$ is group invertible; and in this situation we have

$$
\begin{aligned}
& (a P+b Q)^{\#} \\
& =Z\left(\left(a A_{1}+b B_{1}\right)^{\#} \oplus 0 \oplus 0 \oplus 0\right) Z^{-1}+\frac{1}{a} Z\left(0 \oplus A_{2}^{-1} \oplus 0 \oplus 0\right) Z^{-1}+ \\
& \quad+\frac{1}{b} Z\left(0 \oplus 0 \oplus B_{2}^{-1} \oplus 0\right) Z^{-1} \\
& \quad\left(a P Q Q^{\#}+b Q P P^{\#}\right)^{\#}+\frac{1}{a} P^{\#} Q^{\pi}+\frac{1}{b} Q^{\#} P^{\pi} .
\end{aligned}
$$

The proof is completed.

Acknowledgment. The authors wish thank the referee for his/her helpful comments and for providing some useful references. 


\section{REFERENCES}

[1] A. Ben-Israel and T.N.E. Greville. Generalized Inverses: Theory and Applications, 2nd Edition. Springer-Verlag, New York, 2003.

[2] J. Benítez, X. Liu, and T. Zhu. Nonsingularity and group invertibility of linear combinations of two k-potent matrices. Linear Multilinear Algebra, 58:1023-1035, 2010.

[3] C. Cao. Some results of group inverses for partitioned matrices over skew fields. Heilongjiang Daxue Ziran Kexue Xuebao (Chinese), 18:5-7, 2001.

[4] N. Castro-González, E. Dopazo, and M.F. Martínez-Serrano. On the Drazin inverse of the sum of two operators and its application to operator matrices. J. Math. Anal. Appl., 350:207-215, 2009.

[5] N. Castro-González and J.Y. Vélez-Cerrada. On the perturbation of the group generalized inverse for a class of bounded operators in Banach spaces. J. Math. Anal. Appl., 341:1213$1223,2008$.

[6] D.S. Cvetković-Ilić and C.Y Deng. Some results on the Drazin invertibility and idempotents. J. Math. Anal. Appl., 359:731-738, 2009.

[7] D.S. Cvetković-Ilić, D.S. Djordjević, and Y. Wei. Additive results for the generalized Drazin inverse in a Banach algebra. Linear Algebra Appl., 418:53-61, 2006.

[8] C.Y. Deng. The Drazin inverses of sum and difference of idempotents. Linear Algebra Appl., 430:1282-1291, 2009

[9] C.Y. Deng and Y. Wei. New additive results for the generalized Drazin inverse. J. Math. Anal. Appl., 370:313-321, 2010.

[10] D.S. Djordjević and Y. Wei, Additive results for the generalized Drazin inverse. J. Aust. Math. Soc., 73:115-125, 2002.

[11] R.E. Hartwig, G.R. Wang, and Y. Wei. Some additive results on Drazin inverse. Linear Algebra Appl., 322:207-217, 2001.

[12] X. Liu, L. Wu, and Y. Yu. The group inverse of the combinations of two idempotent matrices. Linear Multilinear Algebra, 59:101-115, 2011.

[13] C.D. Meyer. Matrix Analysis and Applied Linear Algebra. Society for Industrial and Applied Mathematics (SIAM), Philadelphia, 2000.

[14] Y. Wei and C.Y. Deng. A note on additive results for the Drazin inverse. Linear Multilinear Algebra, to appear, 2011. DOI: 10.1080/03081087.2010.496110. 